\title{
A Note on Degenerate Variational Problems with Linear Growth
}

\author{
M. Bildhauer
}

\begin{abstract}
Given a class of strictly convex and smooth integrands $f$ with linear growth, we consider the minimization problem $\int_{\Omega} f(\nabla u) d x \rightarrow$ min and the dual problem with maximizer $\sigma$. Although degenerate problems are studied, the validity of the classical duality relation is proved in the following sense: there exists a generalized minimizer $u^{*} \in B V\left(\Omega ; \mathbb{R}^{N}\right)$ of the original problem such that $\sigma(x)=\nabla f\left(\nabla^{a} u^{*}\right)$ holds almost everywhere, where $\nabla^{a} u^{*}$ denotes the absolutely continuous part of $\nabla u^{*}$ with respect to the Lebesgue measure. In particular, this relation is also true in regions of degeneracy, i.e. at points $x$ such that $D^{2} f\left(\nabla^{a} u^{*}(x)\right)=0$. As an application, we can improve the known regularity results for the dual solution.
\end{abstract}

Keywords: Degenerate problems, linear growth, duality, regularity

AMS subject classification: $49 \mathrm{~N} 15,49 \mathrm{~N} 60$

\section{Introduction}

The minimization problem

$(\mathcal{P}) \quad J(u)=\int_{\Omega} f(\nabla u) d x \rightarrow \min$ in $u_{0}+\stackrel{\circ}{W}_{1}^{1}\left(\Omega ; \mathbb{R}^{N}\right)$

with integrand $f$ of linear growth is in general not solvable, even if $f$ is strictly convex and smooth. One way to overcome this difficulty is to pass to the dual problem which admits a unique (see [2]) solution $\sigma$. This solution may be interpreted for example as the normal to a minimal surface (see [5]) in the case $f(P)=\sqrt{1+|P|^{2}}$, or the stress tensor in the theory of plasticity as studied in a series of papers by Seregin (see [6] for an exhaustive list of references and a precise definition of the energy density for this particular case). The dual problem reads as

$\left(\mathcal{P}^{*}\right) \quad R(\tau):=\inf _{u \in u_{0}+\stackrel{\circ}{W}_{1}^{1}\left(\Omega ; \mathbb{R}^{N}\right)} l(u, \tau) \rightarrow \max$ in $L^{\infty}\left(\Omega ; \mathbb{R}^{n N}\right)$.

If the standard scalar product in $\mathbb{R}^{n N}$ is denoted by $P: Q$, then the Lagrangian $l(u, \tau)$ and the conjugate function $f^{*}$ are by definition

$$
\begin{aligned}
& l(u, \tau)=\int_{\Omega} \tau: \nabla u d x-\int_{\Omega} f^{*}(\tau) d x,(u, \tau) \in\left\{u_{0}+\stackrel{\circ}{W}_{1}^{1}\left(\Omega ; \mathbb{R}^{N}\right)\right\} \times L^{\infty}\left(\Omega ; \mathbb{R}^{n N}\right) \\
& f^{*}(Q)=\sup _{P \in \mathbb{R}^{n N}}\{P: Q-f(P)\}, Q \in \mathbb{R}^{n N} .
\end{aligned}
$$

M. Bildhauer: FR 6.1 Math., Univ. des Saarlandes, PF 1511 50, D-66041 Saarbrücken bibi@math.uni-sb.de 
Problems $(\mathcal{P})$ and $\left(\mathcal{P}^{*}\right)$ are related via (see [5])

$$
\inf \left\{J(u): u \in u_{0}+\stackrel{\circ}{W}_{1}^{1}\left(\Omega ; \mathbb{R}^{N}\right)\right\}=\sup \left\{R(\tau): \tau \in L^{\infty}\left(\Omega ; \mathbb{R}^{n N}\right)\right\}
$$

and - if we assume the existence of regular solutions $u^{*}$ and $\sigma$ of problems $(\mathcal{P})$ and $\left(\mathcal{P}^{*}\right)$, respectively - by the duality relation

$$
\sigma(x)=\nabla f\left(\nabla u^{*}(x)\right) .
$$

A second way to handle the lack of existence is to look for a generalized minimizer of the original problem in $B V\left(\Omega ; \mathbb{R}^{N}\right)$, the space of functions with bounded variation. If we now denote by $u^{*}$ a generalized $B V$-minimizer of problem $(\mathcal{P})$ in the sense that $u^{*} \in \mathcal{M}$,

$$
\mathcal{M}=\left\{\begin{array}{l|l}
u \in B V\left(\Omega ; \mathbb{R}^{N}\right) & \begin{array}{l}
u \text { is the } L^{1} \text {-limit of a } J \text {-minimizing } \\
\text { sequence from } u_{0}+\stackrel{\circ}{W}_{1}^{1}\left(\Omega ; \mathbb{R}^{N}\right)
\end{array}
\end{array}\right\}
$$

then (1.1) becomes

$$
\sigma(x)=\nabla f\left(\nabla u^{*}(x)\right) \quad \text { for all } x \in \Omega_{u^{*}}
$$

where $\Omega_{u^{*}} \subset \Omega$ is the open set of all $u^{*}$-regular points, i.e. $u^{*}$ is of class $C^{1, \alpha}$ in some neighbourhood of $x \in \Omega_{u^{*}}$. Equation (1.2) is established in [3] where some special generalized minimizer is fixed. With this information arbitrary solutions $u^{*} \in \mathcal{M}$ are handled using the relaxed minimax inequality (see, for instance, [4, 12]).

If we assume $D^{2} f(P)>0$ for all $P$, then using the regularity results of [1] it is proved in [3] that $u^{*}$ is regular on an open set of full measure, hence by (1.2) partial $C^{0, \alpha}$-regularity in the usual sense is ensured for $\sigma$.

If degenerate problems are studied, i.e. under the weaker assumption $D^{2} f \geq 0$, then the approach outlined above in general does not lead to satisfying results. Let us sketch the two main problems by considering a prominent example (compare [7]):

$$
f(P)=\left(1+|P|^{k}\right)^{\frac{1}{k}} \quad(k>2) .
$$

On one hand, (1.2) is a quite vague statement since the regular set $\Omega_{u^{*}}$ may be very small. On the other hand, and this is even more restrictive since we are interested in regularity results for $\sigma$, partial Hölder continuity of $\sigma$ for the integrand (1.3) at hand follows a priori only on $\left[D^{2} f\left(\nabla u^{*}(x)\right)>0\right]=\left[\nabla u^{*}(x) \neq 0\right]$. However, an intrinsic theorem should be formulated in terms of $\sigma$, i.e. the domain of partial regularity is expected to be

$$
[\sigma(x) \neq 0] \supset\left[\nabla^{a} u^{*}(x) \neq 0\right]
$$

where the inclusion follows on account of (1.2) and of course is meant modulo sets of measure zero. Here, to be a little bit more precise, $\nabla^{a} u^{*}$ denotes the absolute continuous part of $\nabla u^{*}$ with respect to the Lebesgue-measure and $\nabla^{s} u^{*}$ will be used as the symbol for the singular part.

In our paper, a generalization of the classical duality relation (1.1) is established for almost all $x \in \Omega$ : we leave the regularity of $u^{*}$ as starting point and prove by arguments 
from measure theory and by an approximation Lemma of [3] that there is a generalized minimizer $u^{*} \in B V\left(\Omega ; \mathbb{R}^{N}\right)$ of Problem $(\mathcal{P})$ such that $\sigma(x)=\nabla f\left(\nabla^{a} u^{*}(x)\right)$ for almost all $x \in \Omega$ where the degenerate situation $D^{2} f \geq 0$ also is covered (compare Assumption 2.1). Coming back to the above example, we see that in fact equality (again modulo sets of measure zero) holds in (1.4) and analogous results of course are true in the case of more general degenerate integrands. As an application, an intrinsic regularity theorem for the dual solution $\sigma$ can be formulated just in terms of $\sigma$ and the data, independent of $u^{*}$ (compare Corollary 4.2 for details).

\section{Preliminaries}

Throughout this paper $\Omega \subset \mathbb{R}^{n}$ is assumed to be a bounded Lipschitz domain and the integrand $f$ under consideration satisfies

Assumption 2.1. The function $f$ is smooth, strictly convex and of linear growth in the following sense:

(i) $f \in C^{2}\left(\mathbb{R}^{n N}, \mathbb{R}\right)$.

(ii) $f(P)>0$ for all $P \in \mathbb{R}^{n N}, P \neq 0$, and $f(0)=0$.

(iii) $f((1-\lambda) P+\lambda Q)<(1-\lambda) f(P)+\lambda f(Q)$ for all $P \neq Q \in \mathbb{R}^{n N}$ and for all $0<\lambda<1$. Suppose further that there is a real number $M$ such that, for all $P$ and $Q \in \mathbb{R}^{n N}, 0 \leq D^{2} f(P)(Q, Q) \leq M \frac{1}{\sqrt{1+|P|^{2}}}|Q|^{2}$ where, moreover, strict inequality on the left-hand side is assumed if $P, Q \neq 0$.

(iv) There is a real number $A$ such that $|\nabla f(P)| \leq A$ for all $P \in \mathbb{R}^{n N}$.

(v) For numbers $a>0$ and $b \in \mathbb{R}$ we have $f(P) \geq a|P|+b$ for all $P \in \mathbb{R}^{n N}$.

\section{Remark 2.2.}

1. Of course, assumption (ii) is supposed without loss of generality and the model integrand considered in (1.3) satisfies assumption (iii).

2. $\Omega_{u^{*}}$ is a subset of the non-degenerate points satisfying

$$
\lim _{r \rightarrow 0} \frac{1}{\left|B_{r}(x)\right|} \int_{B_{r}(x)}\left|\nabla^{a} u^{*}-P\right| d x+\lim _{r \rightarrow 0} \frac{1}{\left|B_{r}(x)\right|} \int_{B_{r}(x)}\left|\nabla^{s} u^{*}\right|=0
$$

for some matrix $P \in \mathbb{R}^{n N}$ such that $D^{2} f(P)>0$ (see $[1,3]$ ). Hence we have $\nabla^{a} u^{*}=0$ almost everywhere on the complement $\Omega_{u^{*}}^{c}$ of $\Omega_{u^{*}}$. However, this provides no results at all because no topological information on $\Omega_{u^{*}}^{c}$ is available. Moreover, the singular part $\nabla^{s} u^{*}$ is not necessarily vanishing on $\Omega_{u^{*}}^{c}$.

Notice that by strict convexity and by the Theorem on Domain Invariance (see, for instance, [9: p. 77/Corollary 3.22]) $\nabla f$ is known to be one-to-one, hence an open mapping and $\nabla f: \mathbb{R}^{n N} \rightarrow \operatorname{Im}(\nabla f):=\nabla f\left(\mathbb{R}^{n N}\right)$ is a homeomorphism (compare [2]).

A powerful tool for the analysis of problem $(\mathcal{P})$ and in consequence of problem $\left(\mathcal{P}^{*}\right)$ is the $\delta$-regularization: assume in the following without loss of generality that the boundary values $u_{0}$ are of class $W_{2}^{1}\left(\Omega ; \mathbb{R}^{N}\right)$ (see [3: Remark 6.3] for a natural extension) and consider for any $\delta \in(0,1)$ the perturbed minimization problem 
$\left(\mathcal{P}_{\delta}\right) \quad J_{\delta}(u)=\frac{\delta}{2} \int_{\Omega}|\nabla u|^{2} d x+\int_{\Omega} f(\nabla u) d x \rightarrow \min$ in $u_{0}+\stackrel{\circ}{W}_{2}^{1}\left(\Omega ; \mathbb{R}^{N}\right)$.

Denote further by $u_{\delta}$ the unique solution of problem $\left(\mathcal{P}_{\delta}\right)$ and let

$$
\sigma_{\delta}:=\delta \nabla u_{\delta}+\nabla f\left(\nabla u_{\delta}\right)
$$

Then the Euler equation for problem $\left(\mathcal{P}_{\delta}\right)$ reads as

$$
\int_{\Omega} \sigma_{\delta}: \nabla \varphi d x=0 \quad \text { for all } \varphi \in \stackrel{\circ}{W}_{2}^{1}\left(\Omega ; \mathbb{R}^{N}\right)
$$

Moreover, there is a number $c>0$ satisfying $J_{\delta}\left(u_{\delta}\right) \leq J_{\delta}\left(u_{0}\right) \leq J_{1}\left(u_{0}\right) \leq c$ and it follows immediately that $\sigma_{\delta} \rightarrow: \sigma$ in $L^{2}\left(\Omega ; \mathbb{R}^{N}\right)$ as $\delta \rightarrow 0$. Next, following the ideas of Seregin [11], it is proved in [3] (see Lemma 3.1) that $\sigma$ maximizes the dual variational problem $\left(\mathcal{P}^{*}\right)$. The proof also shows that $u_{\delta}$ is a $J$-minimizing sequence and that

$$
\delta \int_{\Omega}\left|\nabla u_{\delta}\right|^{2} d x \rightarrow 0 \quad \text { as } \delta \rightarrow 0
$$

Finally, on account of $(2.2)$, it is not hard to prove that (again see [3, $10-12]$ )

$$
\left\|\sigma_{\delta}\right\|_{W_{2}^{1}\left(\tilde{\Omega} ; \mathbb{R}^{n N}\right)} \leq c(\tilde{\Omega}) \quad \text { for all open subsets } \tilde{\Omega} \Subset \Omega
$$

Hence, passing to a subsequence (which is not relabelled), we may assume by (2.3) (2.4) that, for a.a. $x \in \Omega$,

$$
\begin{aligned}
\sigma_{\delta}(x) & \rightarrow \sigma(x) \\
\delta \nabla u_{\delta}(x) & \rightarrow 0 .
\end{aligned}
$$

Remark 2.3. Having established (2.4) and thus $(2.5)_{1}$, we make no further use of the upper bound for $D^{2} f$ stated in Assumption 2.1/(iii).

Passing to another subsequence and observing that $J_{\delta}\left(u_{\delta}\right)$ is uniformly bounded, a $L^{1}$-cluster point $u^{*}$ of $u_{\delta}$ is fixed in the following: $u_{\delta} \stackrel{L^{1}}{\longrightarrow} u^{*} \in B V\left(\Omega ; \mathbb{R}^{n N}\right)$ as $\delta \rightarrow 0$.

\section{Main theorem}

With this notation, in particular with subsequences $\left\{u_{\delta}\right\}$ and $\left\{\sigma_{\delta}\right\}$ given as above, our main theorem reads as follows.

Theorem 3.1. The unique solution $\sigma$ of the dual problem $\left(\mathcal{P}^{*}\right)$ satisfies $\sigma(x)=$ $\nabla f\left(\nabla^{a} u^{*}(x)\right)$ for almost all $x \in \Omega$.

Remark 3.2. It remains an open question whether $\sigma=\nabla f\left(\nabla^{a} u\right)$ holds for any generalized minimizer $u \in \mathcal{M}$.

The proof of Theorem 3.1 requires the construction of "large" sets of uniform convergence according to 
Proposition 3.3. There is a measurable function $v: \Omega \rightarrow \mathbb{R}^{n N}$, and for any $\varepsilon>0$ there is a compact set $K \Subset \Omega$ such that:

(i) $\sigma_{\delta} \rightrightarrows \sigma$ on $K, \sigma(x) \notin \partial \operatorname{Im}(\nabla f)$ for all $x \in K$.

(ii) $\delta \nabla u_{\delta} \rightrightarrows 0$ on $K$.

(iii) $\nabla u_{\delta} \rightrightarrows v$ on $K$.

(iv) The restriction of $v$ on $K$ is a continuous function.

(v) $|\Omega-K|<\varepsilon$.

Remark 3.4 In the following it is obvious that we can restrict to the consideration of Lebesgue points of $\sigma$ and $\nabla u^{*}$, respectively. This is always assumed as a general hypothesis.

Proof of Proposition 3.3 Let us first define

$$
v_{j}^{i}(x)=\limsup _{\delta \rightarrow 0} \frac{\partial}{\partial x_{j}} u_{\delta}^{i}(x) \quad(i \in\{1, \ldots, N\}, j \in\{1, \ldots, n\})
$$

which by definition is a measurable function with values in $\overline{\mathbb{R}}$. Now fix $\varepsilon>0$. The uniform convergence stated in (i) and (ii) on a compact set $\widetilde{K} \Subset \Omega$ with $|\Omega-\widetilde{K}|<\frac{\varepsilon}{2}$ follows on account of (2.5) and Egoroff's theorem. Setting $N=\{x \in \Omega: \sigma(x) \in$ $\partial \operatorname{Im}(\nabla f)\}$ it was proved in $[2]$ that $|N|=0$. So, choose an open set $U \supset N$ with $|U|<\frac{\varepsilon}{2}$. Then $K:=\widetilde{K}-U$ is a compact set such that (i) and (ii) are true and in addition $K$ satisfies (v).

Next observe that we have on $K$ as $\delta \rightarrow 0$

$$
\nabla f\left(\nabla u_{\delta}(x)\right)=\sigma_{\delta}(x)-\delta \nabla u_{\delta}(x) \rightarrow \sigma(x)
$$

If $x_{0} \in K$ is fixed, then $\sigma\left(x_{0}\right) \notin \partial \operatorname{Im}(\nabla f)$ implies that there is a constant $\rho=\rho\left(x_{0}\right)$ such that for all $\delta$ sufficiently small $\operatorname{dist}\left(\nabla f\left(\nabla u_{\delta}\left(x_{0}\right)\right), \partial \operatorname{Im}(\nabla f)\right) \geq \rho$. In other words, we have for all $\delta$ sufficiently small

$$
\nabla f\left(\nabla u_{\delta}\left(x_{0}\right)\right) \in C:=\{Q \in \operatorname{Im}(\nabla f): \operatorname{dist}(Q, \partial \operatorname{Im}(\nabla f)) \geq \rho\}
$$

Since $C$ is compact and since $\nabla f$ is a homeomorphism, $(\nabla f)^{-1}(C)$ is compact, in particular $(\nabla f)^{-1}(C)$ is bounded and as a consequence

$$
\limsup _{\delta \rightarrow 0}\left|\nabla u_{\delta}(x)\right|<\infty \quad \text { for all } x \in K
$$

With (3.1) and (3.2), the pointwise convergence of $\nabla u_{\delta}(x)$ on $K$ is obtained since $\nabla f$ is one-to-one. Egoroff's theorem then establishes (iii) on a "large" compact set $\widetilde{K} \subset K$, without loss of generality on $K$. The proof of (iv) is an application of Lusin's theorem and the proposition follows

Given Proposition 3.3, we now come to the 
Proof of Theorem 3.1. Fix $\varepsilon>0$ and choose $K$ according to the proposition. Since $K$ is measurable, the Lebesgue-Besicovitch differentiation theorem yields

$$
\lim _{r \rightarrow 0} \frac{\left|B_{r}(x) \cap K\right|}{\left|B_{r}(x)\right|}=1 \quad \text { for a.a. } x \in K .
$$

It is also known that for almost all $x \in \Omega$ there exists a matrix $P \in \mathbb{R}^{n N}$ such that

$$
\begin{aligned}
& \lim _{r \rightarrow 0} \frac{1}{\left|B_{r}(x)\right|} \int_{B_{r}(x)}\left|\nabla u^{*}-P\right| \\
:= & \lim _{r \rightarrow 0} \frac{1}{\left|B_{r}(x)\right|} \int_{B_{r}(x)}\left|\nabla^{a} u^{*}-P\right| d x+\lim _{r \rightarrow 0} \frac{1}{\left|B_{r}(x)\right|} \int_{B_{r}(x)}\left|\nabla^{s} u^{*}\right| d x \\
= & 0 .
\end{aligned}
$$

Let us first consider the case $P \neq 0$, i.e. $x$ is a non-degenerate point. Going through the lines of [3] we observe that the duality relation as claimed in the theorem holds in this case (see, in particular, [3: Formula (6.6)]). Thus we have to study the case

$$
\lim _{r \rightarrow 0} \frac{1}{\left|B_{r}(x)\right|} \int_{B_{r}(x)}\left|\nabla u^{*}\right|=0 .
$$

Observe that (3.4) implies

$$
\lim _{r \rightarrow 0} \frac{1}{\left|B_{r}(x)\right|} \int_{B_{r}(x)} f\left(\nabla^{a} u^{*}\right) d x=0 .
$$

In fact, on account of the continuity of $f$ and since $f(0)=0$, we may fix a real number $\lambda>0$ and find $\kappa>0$ such that $|P|<\kappa$ implies $f(P)<\lambda$. With $\kappa$ as above we obtain

$$
\begin{aligned}
\lim _{r \rightarrow 0} & \frac{1}{\left|B_{r}(x)\right|} \int_{B_{r}(x)} f\left(\nabla^{a} u^{*}\right) d x \\
\leq & \limsup _{r \rightarrow 0} \frac{1}{\left|B_{r}(x)\right|} \int_{B_{r}(x) \cap\left[\left|\nabla^{a} u^{*}\right|<\kappa\right]} f\left(\nabla^{a} u^{*}\right) d x \\
& \quad+\limsup _{r \rightarrow 0} \frac{1}{\left|B_{r}(x)\right|} \int_{B_{r}(x) \cap\left[\left|\nabla^{a} u^{*}\right|>\kappa\right]} f\left(\nabla^{a} u^{*}\right) d x .
\end{aligned}
$$

Here the second term on the right-hand side vanishes by the linear growth of $f$ and by (3.4): for some real numbers $c_{1}, c_{2}>0$ we have

$$
\begin{aligned}
& \limsup _{r \rightarrow 0} \frac{1}{\left|B_{r}(x)\right|} \int_{B_{r}(x) \cap\left[\left|\nabla^{a} u^{*}\right|>\kappa\right]} f\left(\nabla^{a} u^{*}\right) d x \\
& \leq c_{1} \limsup _{r \rightarrow 0} \frac{1}{\left|B_{r}(x)\right|} \int_{B_{r}(x) \cap\left[\left|\nabla^{a} u^{*}\right|>\kappa\right]}\left|\nabla^{a} u^{*}\right| d x \\
& \quad+c_{2} \limsup _{r \rightarrow 0} \frac{1}{\left|B_{r}(x)\right|} \int_{B_{r}(x) \cap\left[\left|\nabla^{a} u^{*}\right|>\kappa\right]} 1 d x \\
& =0 .
\end{aligned}
$$


The remaining term is bounded from above by $\lambda$, which is an arbitrary fixed positive number, thus claim (3.5) is proved.

With (3.3) and (3.4) we define a set $G_{K}$ satisfying $\left|K-G_{K}\right|=0$ as follows:

$$
G_{K}:=\left\{x \in K:(3.3) \text { and (3.4) hold true or } \sigma(x)=\nabla f\left(\nabla^{a} u^{*}(x)\right)\right\} .
$$

Remark 3.5. Let us give a short comment on this definition. Consider the set of all $x \in K$ such that (3.3) holds. Then we have to distinguish between the cases " $P=0$ ", i.e. (3.4) is true, and " $P \neq 0$ ". As already mentioned, in the second case the duality relation is proved in [3], in particular $\left|K-G_{K}\right|=0$.

According to this remark, we fix $\hat{x} \in G_{K}$ satisfying (3.3) and (3.4) and recall the fact that $f$ achieves its absolute minimum at $P=0$, hence $0=\nabla^{a} u^{*}(\hat{x})$ and $0=\nabla f(0)$. Then we claim that

$$
\sigma(\hat{x})=0=\nabla f(0)=\nabla f\left(\nabla^{a} u^{*}(\hat{x})\right)
$$

which immediately yields the theorem by passing to the limit $\varepsilon \rightarrow 0$. To prove (3.6) assume by contradiction that $\sigma(\hat{x}) \neq 0$. We now claim that there is a real number $\gamma=\gamma(\hat{x})>0$ such that for all $\delta$ sufficiently small

$$
\gamma<\left|\nabla u_{\delta}(\hat{x})\right|
$$

To verify (3.7) let $\tau=|\sigma(\hat{x})|$ and choose $\delta_{0}>0$ sufficiently small to obtain $\mid \sigma_{\delta}(\hat{x})-$ $\sigma(\hat{x}) \mid<\frac{\tau}{2}$ and $\left|\delta \nabla u_{\delta}(\hat{x})\right|<\frac{\tau}{4}$ for all $\delta<\delta_{0}$. This gives for all $\delta<\delta_{0}$

$$
\left|\nabla f\left(\nabla u_{\delta}(\hat{x})\right)\right| \geq\left|\sigma_{\delta}(\hat{x})\right|-\left|\delta \nabla u_{\delta}(\hat{x})\right|>\frac{\tau}{4} .
$$

If it is supposed in contradiction to (3.7) that there is a sequence $\delta_{n} \rightarrow 0$ such that $\nabla u_{\delta_{n}}(\hat{x}) \rightarrow 0$ as $n \rightarrow \infty$, then the continuity of $\nabla f$ yields $\nabla f\left(\nabla u_{\delta_{n}}(\hat{x})\right) \rightarrow \nabla f(0)=0$ as $n \rightarrow \infty$ which is excluded by (3.8), hence (3.7) is proved.

By Proposition 3.3/(iii), it also follows that $\gamma \leq|v(\hat{x})|$. Thus, by continuity of $v$ on $K$, there is a real number $\rho_{0}>0$ such that $B_{4 \rho_{0}}(\hat{x}) \Subset \Omega$ and such that, for any $\rho<\rho_{0}$, $\frac{\gamma}{2} \leq|v(x)|$ for all $x \in B_{\rho}(\hat{x}) \cap K$. Finally, setting $\kappa=\frac{\gamma}{4}$ and recalling the uniform convergence stated in Proposition 3.3/(iii), we decrease $\delta_{0}$ - if necessary - and arrive at

$$
\kappa \leq\left|\nabla u_{\delta}(x)\right| \quad \text { for all } x \in B_{\rho}(\hat{x}) \cap K, 0<\rho<\rho_{0}
$$

and for all $\delta<\delta_{0}$.

Remark 3.6. If in the sense of measures

$$
\left|\nabla u_{\delta}\right| \rightarrow\left|\nabla u^{*}\right|
$$

would be known, then the compactness of $\bar{B}_{\rho}(\hat{x}) \cap K$ would imply

$$
\kappa\left|\bar{B}_{\rho}(\hat{x}) \cap K\right| \leq \limsup _{\delta \rightarrow 0}\left|\nabla u_{\delta}\right|\left(\bar{B}_{\rho}(\hat{x}) \cap K\right) \leq\left|\nabla u^{*}\right|\left(\bar{B}_{\rho}(\hat{x}) \cap K\right)
$$

and passing to the limit $\rho \rightarrow 0$ a contradiction would follow from (3.4) and from density relation $(3.3)$.

Hence we have to establish an appropriate substitute for (3.10), where we use the "minimality" of $\left\{u_{\delta}\right\}$ as additional information. To this purpose let us introduce for any Lipschitz domain $\hat{\Omega} \subset \Omega$ the following relaxation. 
Definition 3.7. For all $w \in B V\left(\widehat{\Omega} ; \mathbb{R}^{N}\right)$ the functional $\hat{J}(w ; \widehat{\Omega})$ is given by

$$
\hat{J}(w ; \widehat{\Omega})=\inf \left\{\liminf _{k \rightarrow \infty} J\left(w_{k}\right): w_{k} \in C^{1}\left(\widehat{\Omega} ; \mathbb{R}^{N}\right), w_{k} \rightarrow w \text { in } L_{l o c}^{1}\left(\hat{\Omega} ; \mathbb{R}^{N}\right)\right\}
$$

Moreover, a representation formula due to Goffman and Serrin is needed (see [8]).

Proposition 3.8. The representation formula

$$
\hat{J}(u, \widehat{\Omega})=\int_{\widehat{\Omega}} f\left(\nabla^{a} u\right) d x+\int_{\widehat{\Omega}} f_{\infty}\left(\frac{\nabla^{s} u}{\left|\nabla^{s} u\right|}\right) d\left|\nabla^{s} u\right|
$$

is true for all $u \in B V\left(\widehat{\Omega} ; \mathbb{R}^{N}\right)$, where $f_{\infty}$ is the recession function of $f$ defined by $f_{\infty}(X)=\limsup _{t \rightarrow+\infty} \frac{f(t X)}{t}$. As usual, the absolutely continuous part of $\nabla u$ with respect to the Lebesgue measure is denoted by $\nabla^{a} u$, the singular part by $\nabla^{s} u$ and $\nabla^{s} u /\left|\nabla^{s} u\right|$ is the symbol for the Radon-Nikodym derivative.

With this notation the sequence $\left\{u_{\delta}\right\}$ is modified as outlined in [3]. There it is proved that we may choose for almost any $\rho$ as above a sequence $\left\{w_{m}\right\} \subset u_{0}+\stackrel{\circ}{W}_{1}^{1}\left(\Omega ; \mathbb{R}^{N}\right)$, $\widetilde{w}_{m}:=w_{m \mid B_{2 \rho}(\hat{x})}$, satisfying the following conditions:

(i) $w_{m} \rightarrow u^{*}$ in $L^{1}\left(\Omega ; \mathbb{R}^{N}\right)$ as $m \rightarrow \infty$.

(ii) $w_{m \mid \partial B_{2 \rho}(\hat{x})}=u_{\mid \partial B_{2 \rho}(\hat{x})}^{*}$.

(iii) $\liminf \inf _{m \rightarrow \infty} I\left(\widetilde{w}_{m}\right)=\inf _{\mathbb{K}} I=\hat{J}\left(u^{*} ; B_{2 \rho}(\hat{x})\right)$.

(iv) $\widetilde{w}_{m \mid B_{\rho}(\hat{x})}=u_{\delta_{m} \mid B_{\rho}(\hat{x})}$.

Here $\left\{u_{\delta_{m}}\right\}$ denotes a subsequence of $\left\{u_{\delta}\right\}$ and we have abbreviated

$$
\begin{aligned}
& I: W_{1}^{1}\left(B_{2 \rho}(\hat{x}) ; \mathbb{R}^{N}\right) \rightarrow \mathbb{R}, \quad I(w)=\int_{B_{2 \rho}(\hat{x})} f(\nabla w) d x \\
& \mathbb{K}=\left\{w \in W_{1}^{1}\left(B_{2 \rho}(\hat{x}) ; \mathbb{R}^{N}\right): w_{\mid \partial B_{2 \rho}(\hat{x})}=u_{\mid \partial B_{2 \rho}(\hat{x})}^{*}\right\}
\end{aligned}
$$

Remark 3.9. Let us also give some comments on this construction: (i) and (ii) are explicitely stated in [3: Lemma 7.1]. If $\left|\nabla u_{\delta}\right| \rightarrow: \mu$ in the sense of measures, then $\rho$ has to be chosen such that $\mu\left(\partial B_{2 \rho}(\hat{x})\right)=0=\left|\nabla u^{*}\right|\left(\partial B_{2 \rho}(\hat{x})\right)$. Assertion (iii) is proved in [3: Formulas (5.1) and (5.2)]. Finally, (iv) is immediately verified following the proof of [3: Lemma 7.1].

Now convexity of $f$ and Assumption 2.1/(ii) imply the existence of a real number $\vartheta>0$ such that $f(P)>\vartheta$ whenever $|P| \geq \kappa$. Hence we deduce from (3.9) and (iv) that $\vartheta \leq f\left(\nabla \widetilde{w}_{m}\right)$ for all $x \in B_{\rho}(\hat{x}) \cap K$ and for all $m \in \mathbb{N}$. This yields (recall $f \geq 0$, see (iii) 
and Proposition 3.8)

$$
\begin{aligned}
& \vartheta \frac{\left|K \cap B_{\rho}(\hat{x})\right|}{\left|B_{2 \rho}(\hat{x})\right|} \\
& \quad \leq \frac{1}{\left|B_{2 \rho}(\hat{x})\right|} \liminf _{m \rightarrow \infty} \int_{B_{2 \rho}(\hat{x})} f\left(\nabla \tilde{w}_{m}\right) d x \\
& \quad=\frac{1}{\left|B_{2 \rho}(\hat{x})\right|} \inf _{\mathbb{K}} I \\
& \quad=\frac{1}{\left|B_{2 \rho}(\hat{x})\right|} \hat{J}\left(u^{*} ; B_{2 \rho}(\hat{x})\right) \\
& \quad=\frac{1}{\left|B_{2 \rho}(\hat{x})\right|} \int_{B_{2 \rho}(\hat{x})} f\left(\nabla^{a} u^{*}\right) d x+\frac{1}{\left|B_{2 \rho}(\hat{x})\right|} \int_{B_{2 \rho}(\hat{x})} f_{\infty}\left(\frac{\nabla^{s} u^{*}}{\left|\nabla^{s} u^{*}\right|}\right) d\left|\nabla^{s} u^{*}\right| .
\end{aligned}
$$

Both sides of (3.11) are independent of $m$ and we may now pass to the limit $\rho \rightarrow 0$. By the density assumption (3.3) it is seen that

$$
\lim _{\rho \rightarrow 0} \vartheta \frac{\left|K \cap B_{\rho}(\hat{x})\right|}{\left|B_{2 \rho}(\hat{x})\right|}=\vartheta 2^{-n}
$$

whereas on account of $(3.4),(3.5)$ and the boundedness of $f_{\infty}$

$$
\lim _{\rho \rightarrow 0}\left\{\frac{1}{\left|B_{2 \rho}(\hat{x})\right|} \int_{B_{2 \rho}(\hat{x})} f\left(\nabla^{a} u^{*}\right) d x+\frac{1}{\left|B_{2 \rho}(\hat{x})\right|} \int_{B_{2 \rho}(\hat{x})} f_{\infty}\left(\frac{\nabla^{s} u^{*}}{\left|\nabla^{s} u^{*}\right|}\right) d\left|\nabla^{s} u^{*}\right|\right\}=0 .
$$

Thus, this together with (3.12) contradicts (3.11) and Theorem 3.1 is proved

\section{Application to regularity theory}

We finish this paper with a short application of Theorem 3.1 to regularity theory: the $u^{*}$ - and the $\sigma$-degenerate sets are identified modulo sets of measure zero and, as a consequence, an intrinsic regularity theorem for $\sigma$ is obtained for the degenerate problems under consideration. To this purpose, consider $u^{*}$ as given above and set

$$
\begin{aligned}
& \Omega_{u^{*}}^{\mathrm{deg}}=\left\{x \in \Omega: \nabla^{a} u^{*}(x)=0\right\} \\
& \Omega_{\sigma}^{\mathrm{deg}}=\{x \in \Omega: \sigma(x)=\nabla f(0)=0\} .
\end{aligned}
$$

Then $\Omega_{u^{*}}^{\text {deg }}$ and $\Omega_{\sigma}^{\text {deg }}$ are well defined on the complements of sets of measure zero. For a more precise definition one has to consider Lebesgue points of $\sigma, \nabla^{a} u^{*}$ respectively where the singular part $\nabla^{s} u^{*}$ should vanish. Since $\nabla f$ is one-to-one, Theorem 3.1 implies:

Corollary 4.1. With the above assumptions there exists a generalized minimizer $u^{*} \in \mathcal{M}$ such that $\left|\Omega_{u^{*}}^{\mathrm{deg}}-\Omega_{\sigma}^{\mathrm{deg}}\right|=\left|\Omega_{\sigma}^{\mathrm{deg}}-\Omega_{u^{*}}^{\mathrm{deg}}\right|=0$. 
On the other hand, as proved in [3], $u^{*}$ is on almost any ball $B_{R}\left(x_{0}\right) \Subset \Omega$ a local $\hat{J}$-minimizer and the regularity results of [1] imply the existence of an open set $\Omega^{\text {reg }} \subset$ $\Omega-\Omega_{u^{*}}^{\text {deg }}$ such that, for any $0<\alpha<1, u^{*} \in C^{1, \alpha}\left(\Omega^{\text {reg }}, \mathbb{R}^{N}\right)$ and $\left|\left(\Omega-\Omega_{u^{*}}^{\text {deg }}\right)-\Omega^{\text {reg }}\right|=0$. By Theorem 3.1 (in fact (1.2) is sufficient), the dual solution $\sigma$ is known to be of class $C^{0, \alpha}$ on $\Omega^{\text {reg }}$. Now observe that again by $(1.2)$ and since $\nabla f$ is one-to-one the inclusion $\Omega^{\text {reg }} \subset \Omega-\Omega_{\sigma}^{\text {deg }}$ also holds true. Applying Corollary 4.1 we get the following partial regularity result for $\sigma$.

Corollary 4.2. If $f$ is given as above and if $\sigma$ denotes the unique solution of the dual variational problem $\left(\mathcal{P}^{*}\right)$, then there is an open set $\Omega^{\text {reg }} \subset \Omega-\Omega_{\sigma}^{\text {deg }}$ such that, for any $0<\alpha<1, \sigma \in C^{0, \alpha}\left(\Omega^{\text {reg }}, \mathbb{R}^{n N}\right)$ and $\left|\left(\Omega-\Omega_{\sigma}^{\text {deg }}\right)-\Omega^{\text {reg }}\right|=0$.

\section{References}

[1] Anzellotti, G. and M. Giaquinta: Convex functionals and partial regularity. Arch. Rat. Mech. Anal. 102 (1988), $243-272$.

[2] Bildhauer, M.: A uniqueness theorem for the dual problem associated to a variational problem with linear growth. Zap. Nauchn. Sem. St.-Petersburg Odtel. Math. Inst. Steklov (POMI) 271 (2000), $83-91$.

[3] Bildhauer, M. and M. Fuchs: Regularity for dual solutions and for weak cluster points of minimizing sequences of variational problems with linear growth. Zap. Nauchn. Sem. St.Petersburg Odtel. Math. Inst. Steklov (POMI) 259 (1999), 46 - 66.

[4] Bildhauer, M. and M. Fuchs: On a class of variational integrals with linear growth satisfying the condition of $\mu$-ellipticity. Preprint. Bonn: University/SFB 256, Preprint no. 681 (2000).

[5] Ekeland, I. and R. Temam: Convex Analysis and Variational Problems. Amsterdam: North-Holland 1976.

[6] Fuchs, M. and G. Seregin: Variational Methods for Problems from Plasticity Theory and for Generalized Newtonian Fluids. Lect. Notes Math 1749 (2000).

[7] Giaquinta, G. M. and J. Souček: Functionals with linear growth in the calculus of variations, Parts I and II. Comment. Math. Univ. Carolinae 20 (1979), 143 - 172.

[8] Goffman, C. and J. Serrin: Sublinear functions of measures and variational integrals. Duke Math. J. 31 (1964), 159 - 168.

[9] Schwartz, J. T.: Nonlinear Functional Analysis. New York et al.: Gordon and Breach Sci. Publ. 1969.

[10] Seregin, G.: Differentiability of local extremals of variational problems in the mechanics of perfect elastoplastic media (in Russian). Diff. Uravn. 23 (11) (1987), 1981 - 1991: Engl. transl. in: Diff. Equ. 23 (1987), 1349 - 1358.

[11] Seregin, G.: Differential properties of solutions of variational problems for functionals with linear growth. Problemy Mat. Anal. (Leningr. Univ. LGU) 11 (1990), 51 - 79; Engl. transl. in J. Soviet Math. 64 (1993), 1256 - 1277.

[12] Seregin, G.: Twodimensional variational problems in plasticity theory (in Russian). Izv. Russ. Acad. Sci. 60 (1996), 175 - 210; Engl. transl. in Izvestiya Math. 60 (1996), 179 216. 
Received 13.09.1999; in revised version 04.04.2001 